\title{
Smokescreen: a targeted genotyping array for addiction research
}

\author{
James W. Baurley ${ }^{*}{ }^{\dagger}$, Christopher K. Edlund ${ }^{\dagger}$, Carissa I. Pardamean, David V. Conti and Andrew W. Bergen
}

\begin{abstract}
Background: Addictive disorders are a class of chronic, relapsing mental disorders that are responsible for increased risk of mental and medical disorders and represent the largest, potentially modifiable cause of death. Tobacco dependence is associated with increased risk of disease and premature death. While tobacco control efforts and therapeutic interventions have made good progress in reducing smoking prevalence, challenges remain in optimizing their effectiveness based on patient characteristics, including genetic variation. In order to maximize collaborative efforts to advance addiction research, we have developed a genotyping array called Smokescreen. This custom array builds upon previous work in the analyses of human genetic variation, the genetics of addiction, drug metabolism, and response to therapy, with an emphasis on smoking and nicotine addiction.
\end{abstract}

Results: The Smokescreen genotyping array includes 646,247 markers in 23 categories. The array design covers genomewide common variation (65.67, 82.37, and $90.72 \%$ in African (YRI), East Asian (ASN), and European (EUR) respectively); most of the variation with a minor allele frequency $\geq 0.01$ in 1014 addiction genes $(85.16,89.51$, and $90.49 \%$ for YRI, ASN, and EUR respectively); and nearly all variation from the 1000 Genomes Project Phase 1, NHLBI GO Exome Sequencing Project and HapMap databases in the regions related to smoking behavior and nicotine metabolism: CHRNA5-CHRNA3CHRNB4 and CYP2A6-CYP2B6. Of the 636 pilot DNA samples derived from blood or cell line biospecimens that were genotyped on the array, 622 (97.80 \%) passed quality control. In passing samples, $90.08 \%$ of markers passed quality control. The genotype reproducibility in 25 replicate pairs was $99.94 \%$. For 137 samples that overlapped with HapMap2 release 24 , the genotype concordance was $99.76 \%$. In a genome-wide association analysis of the nicotine metabolite ratio in 315 individuals participating in nicotine metabolism laboratory studies, we identified genome-wide significant variants in the CYP2A6 region ( $\min p=9.10 \mathrm{E}-15)$.

Conclusions: We developed a comprehensive genotyping array for addiction research and demonstrated its analytic validity and utility through pilot genotyping of HapMap and study samples. This array allows researchers to perform genome-wide, candidate gene, and pathway-based association analyses of addiction, tobacco-use, treatment response, comorbidities, and associated diseases in a standardized, high-throughput platform.

Keywords: Addiction, Nicotine dependence, Nicotine metabolism, Pharmacogenomics, Smoking cessation, Genomewide association study, Bioinformatics, Biomarkers

\section{Background}

Addictive disorders represent debilitating conditions that result in productivity loss, and an increased risk for associated mental disorders as well as infectious, metabolic, proliferative, respiratory, and vascular diseases [1]. Addictions encompass substance-use disorders and compulsive behaviors [2]. The heritabilities of substance-use disorders are consistently found to be $\sim 50 \%$ [3] with the lowest

\footnotetext{
*Correspondence: baurley@biorealmresearch.com

${ }^{\dagger}$ Equal contributors

BioRealm LLC, 6101 W. Centinela Ave., Suite 270, Culver City, CA 90230-6359, USA
}

heritability for hallucinogen and highest for cocaine use disorders, respectively [4]. The $\sim 2: 1$ monozygotic:dizygotic twin concordance ratios for many substance-use disorders support additive genetic effects and multiple loci [4]. Estimates for the influence of genome-wide common variants on nicotine and alcohol dependence, and illicit drug use traits are $\sim 30-36 \%[5,6]$, representing most of the estimated heritability. There is evidence for shared genetic influence across multiple substance-use disorders $[6,7]$.

Among all substance-use disorders, smoking is the leading cause of preventable death in the United States 
and is associated with cancer, aerodigestive tract, genitourinary tract, and vascular diseases [8]. Nicotine dependence is most often assessed using measures of cigarette consumption (cigarettes per day, CPD) or dependence (Fagerström Test for Cigarette Dependence, FTCD) [9, 10]. Genetic influences predominate over environmental influences in smoking initiation, but shared environment is important during the adolescent period when the majority of future smokers initiate smoking [11]. Developmental analyses suggest that genetic and environmental factors that influence risk for smoking initiation and consumption are independent in adolescence, but become correlated in emerging adults [12]. Genome-wide meta-analyses of cigarette consumption, dependence, and exposure measures, including linkage [13, 14] and association [15-22] studies, have focused attention on multiple regions, including specific variants in cholinergic, cytochrome oxidase, dopaminergic, and hypoxia response genes.

People who quit smoking reduce their risk of disease and those who quit at an earlier age see the most health benefits [23]. An estimated $69 \%$ of the approximately 45 million smokers in the United States want to stop smoking [24, 25]. Nonetheless, smoking cessation success rates remain discouragingly low; in 2010, only $6.2 \%$ of adult smokers attempting to quit were successful [25]. For those seeking to quit, there are a variety of counseling methods and cessation medications available [26, 27].

Genetics plays a role in smokers' cessation attempts (heritabilities of $\sim 50 \%[28,29]$ ), and response to cessation treatments [30]. Pharmacogenetic analyses of smoking cessation clinical trials suggest that prospective abstinence is affected by loci influencing reward system responses to nicotine and pharmacotherapeutics, nicotine and bupropion metabolism, and varenicline clearance [30]. Analyses of chr15q25.1 SNPs and prospective abstinence by pharmacotherapy have been mixed: null [31-33], reduced in participants randomized to placebo [34], and increased in participants randomized to multiple therapies $[35,36]$. In the largest analysis to date [37], chr15q25.1 smoking-heaviness risk SNPs were found to be associated with reduced abstinence in participants randomized to placebo, and increased abstinence in participants randomized to nicotine replacement therapy (NRT). In contrast, studies on functional variation in the nicotine metabolizing enzyme cytochrome P450 oxidase 2A6 (CYP2A6) $[38,39]$ translate robustly to smoking behaviors $[40,41]$ and prospective abstinence, using either nicotine metabolite [42-45], or genetic [46-48] analyses. In brief, recent findings suggest that slow nicotine metabolizers are less nicotine dependent and have similar quit rates across therapies, while fast nicotine metabolizers are more nicotine dependent and may benefit from combined treatments with NRT, bupropion, or varenicline.
Despite these successes, attempts to individualize therapies using genetics have been limited by inconsistent results. Existing evidence suggests that smoking cessation prediction is influenced by not just genetics, but also by patient characteristics (gender, age of onset, nicotine dependence, and race/ethnicity) and treatment protocol (clinician interaction; type, number and length of counseling sessions; and pharmacotherapy). Biomarkers, specifically the nicotine metabolite ratio (NMR, the ratio of 3'-trans-hydroxycotinine/cotinine), have also been shown to influence pharmacological therapies success [42, 43, 45]. Advancing the understanding of complex relationships among multiple genetic and environmental factors, smoking behavior, nicotine dependence, and treatment outcomes requires large sample sizes. Current clinical studies are challenged by relatively small sample sizes in treatment arms; thus, pooling data across clinical trials and observational studies is essential, but complicated by heterogeneity in trial study designs, genotyping technologies, and genetic marker content.

Motivated by these obstacles, we developed the Smokescreen genotyping array, a research tool for significantly advancing the understanding of addiction and the development of predictive models that can potentially be used to personalize treatment strategies for addiction, including nicotine- and tobacco-related outcomes.

\section{Results}

\section{Array design and coverage estimates}

The final array design included 646,247 markers: 296,038 genome-wide association markers; 255,862 tag SNPs and 17,632 exonic markers in addiction-related gene regions; 11,099 fine-mapping markers in loci related to nicotine metabolism and smoking behavior; and the remaining markers in other categories (Table 1; markers in categories have some overlap). Coverage estimates are provided using the number of variants available through genotype imputation in the 1000 Genomes Project Phase 1 [49] Yoruba in Ibadan, Nigeria (YRI), East Asian (ASN), and European (EUR) populations (Table 2).

\section{Genome-wide association markers}

The array includes 296,038 markers for genome-wide coverage of common genetic variations. The relationship between genome-wide array coverage and the observed correlation of imputed and actual genotypes (obsRSQ) in 1000 Genomes Phase 1 YRI, ASN, and EUR populations is shown in Fig. 1 (left panel). As the threshold on obsRSQ increases ( $x$-axis), fewer variants exceed the threshold, and the coverage decreases ( $y$-axis). The array design achieves good coverage of common variants (MAF $\geq 0.05$, obsRSQ $>0.8$ ): 65.67, 82.37, and $90.72 \%$ in YRI, ASN, and EUR populations respectively, and covers $52.71,71.68$, and $78.17 \%$ respectively of 
Table 1 Smokescreen genotyping array content

\begin{tabular}{|c|c|}
\hline Category & Markers $^{a}$ \\
\hline \multicolumn{2}{|l|}{1014 addiction-related genes } \\
\hline Tag SNPs (MAF $\geq 0.05$ ) & 255862 \\
\hline Exonic markers & 17632 \\
\hline \multicolumn{2}{|l|}{ Genome-wide association markers } \\
\hline Affymetrix’ Axiom ${ }^{\oplus}$ Biobank GWAS grid & 246038 \\
\hline African (YRI) booster panel & 50000 \\
\hline \multicolumn{2}{|l|}{ Fine-mapping of smoking related loci } \\
\hline CHRNA5-CHRNA3-CHRNB4 (552 kb LD block) & 8913 \\
\hline CYP2A6 ( $\pm 20 \mathrm{~kb})$ & 573 \\
\hline CYP2B6 ( $\pm 20 \mathrm{~kb})$ & 1613 \\
\hline \multicolumn{2}{|l|}{ High-value addiction markers } \\
\hline NeuroSNP Project & 4994 \\
\hline $\begin{array}{l}\text { Pharmacogenetics of Nicotine Addiction } \\
\text { Treatment (PNAT) SNP panels }\end{array}$ & 2271 \\
\hline v1.0 Quit Success Score & 12058 \\
\hline Literature search & 1329 \\
\hline \multicolumn{2}{|l|}{ Comorbidity markers } \\
\hline Lung Cancer & 3091 \\
\hline Psychiatric disorders & 1200 \\
\hline $\begin{array}{l}\text { Tobacco smoke constituent update and } \\
\text { metabolic phenotypes }\end{array}$ & 1907 \\
\hline Pulmonary diseases and traits & 7945 \\
\hline Cardiovascular diseases and traits & 2247 \\
\hline \multicolumn{2}{|l|}{ General high-value markers } \\
\hline Pharmacogenomic markers & 2030 \\
\hline NHGRI GWAS Catalog & 7612 \\
\hline eQTLs & 9736 \\
\hline Loss-of-function markers & 4680 \\
\hline Ancestry informative markers (AIMs) & 5545 \\
\hline $\mathrm{HLA} / \mathrm{KIR}$ & 8894 \\
\hline Mitochondrial & 180 \\
\hline Array Total & 646247 \\
\hline
\end{tabular}

${ }^{\mathrm{a}}$ Markers in categories may overlap

variants with $M A F \geq 0.01$. The average obsRSQ for variants with $\mathrm{MAF} \geq 0.05$ were $0.82,0.89$ and 0.93 for YRI, ASN, and EUR respectively, and 0.72, 0.80, and 0.85 for variants with $\mathrm{MAF} \geq 0.01$. These metrics demonstrate the array's suitability for genome-wide analyses and meta analyses based on genotype imputation using 1000 Genomes Project Phase 1 data.

\section{Addiction genes}

The array contains 273,494 markers for dense coverage of 1014 genes $( \pm 20 \mathrm{~kb})$ across populations. The array captures 97.47, 98.09, $98.08 \%$ of common variants $(\mathrm{MAF} \geq 0.05)$ and $63.61 \%, 84.02$, and $82.55 \%$ of MAF $\geq$
Table 2 Counts of imputed SNPs (1000 Genomes Project: Phase 1, March 2012 release)

\begin{tabular}{cccc}
\hline & YRI & ASN & EUR \\
\hline Genome-Wide & & & \\
MAF $\geq 0.01$ & 15263433 & 8091434 & 9213645 \\
MAF $\geq 0.05$ & 9219112 & 5973609 & 6505846 \\
Addiction Genes & & & \\
MAF $\geq 0.01$ & 794696 & 417902 & 476661 \\
MAF $\geq 0.05$ & 474408 & 303991 & 333356 \\
CHRNA5, CHRNA3 and CHRNB4 & & \\
MAF $\geq 0.01$ & 3377 & 2377 & 1922 \\
MAF $\geq 0.05$ & 2121 & 1862 & 1519 \\
CYP2A6 and CYP2B6 & & & \\
MAF $\geq 0.01$ & 2886 & 2679 & 2696 \\
MAF $\geq 0.05$ & 2157 & 2043 & 2136 \\
\hline
\end{tabular}

0.01 variants in YRI, ASN, and EUR populations, respectively, directly through linkage disequilibrium (pairwise $r^{2} \geq 0.8$ ). The fraction of variants covered by genotype imputation in these gene regions for YRI, ASN, and EUR populations is shown in Fig. 1 (right panel). Consequently, the array has outstanding imputation coverage (98.72, 99.31, and $99.43 \%)$ of common variants and excellent coverage of MAF $\geq 0.01$ variants: $85.16,89.51$, and $90.49 \%$ for YRI, ASN, and EUR respectively (obsRSQ $\geq 0.8$ ). In the same order, average obsRSQ of all variants within the addiction gene regions were $0.98,0.99$, and 0.99 for common variants, and $0.91,0.92$, and 0.94 for MAF $\geq 0.01$ variants, indicating that imputation works well for most variants in these regions. The list of 1014 genes and each region's imputation metrics are provided in Additional file 1.

\section{Fine-mapping}

Eight thousand, nine hundred and fourty-eight SNPs and indels (average of 1 marker per 62 base pairs) were selected for the $552 \mathrm{~kb}$ LD block encompasing the chr15q25.1 nicotinic acetylcholine receptor (nAChR) gene cluster (CHRNA5, CHRNA3 and CHRNB4). For CYP2A6 ( $\pm 20 \mathrm{~kb})$, 612 markers were selected with an average of 1 marker every 75 base pairs. For CYP2B6 $( \pm 20 \mathrm{~kb}), 1628$ markers were selected with 1 marker per 45 base pairs on average. As expected, the imputed coverage and average obsRSQ was greater than $99 \%$ in the 1000 Genomes Project Phase 1 YRI, ASN, and EUR populations, regardless of minor allele frequency. Additional markers were selected to capture variation in the surrounding region, including EGLN2, CYP2A7, CYP2G1P, and CYP2B7P1.

\section{Additional content}

Additional markers were included in the design for compatibility with consortium-developed arrays and most recent findings (Table 1). The array contains: 2271 markers 


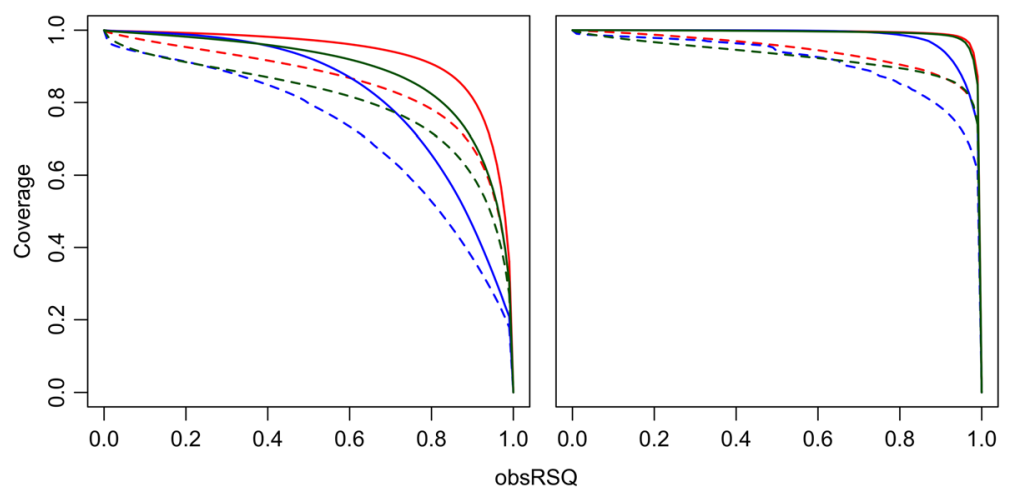

Fig. 1 Smokescreen coverage estimates by increasing observed imputation $r^{2}$ thresholds: genome-wide and addiction genes. Left panel: Genome-wide coverage. Right panel: Addiction genes coverage. Solid line: MAF $\geq 0.05$. Dashed line: MAF $\geq 0.01$. Red: EUR. Blue: YRI. Green: ASN. Observed imputation $r^{2}$ (obsRSQ) is the correlation between imputed (continuous) genotype dosage and the measured genotype from the 1000 Genomes Project. The proportion of 1000 Genome Project Phase 1 variants with an obsRSQ above the threshold on the $x$-axis is represented on the $y$-axis. The average obsRSQ differs by race/ethnicity and by array content categories (e.g., genome-wide versus addiction genes). The coverage (fraction of variants with obsRSQ above the threshold) decreases as the obsRSQ threshold increases. A typical threshold used in evaluating array coverage is 0.80

from the candidate gene/pathway arrays developed and used by the Pharmacogenetics of Nicotine Addiction Treatment (PNAT) research program [31, 37]; 3091 markers from a lung cancer meta-GWAS [50]; 1200 markers related to psychiatric comorbidities from the Psychiatric Genetics Consortium [51]; and 7956 and 2247 markers for pulmonary and cardiovascular phenotypes respectively from the UK Biobank Axiom Array [52].

The array also includes: 1329 markers related to addiction identified in recent literature from NIDA Genetics Consortium investigators; 12,058 markers used in the smoking cessation v1.0 Quit Success Score biomarker [53]; 2030 pharmacogenomic markers related to absorption, distribution, metabolism, and excretion (ADME); and 7612 markers identified in previous genome-wide association studies or addiction and related diseases [54]. The array includes a panel of 5525 ancestry informative markers (AIMs) for ancestry estimation and evaluation of population substructure.

\section{Genotyping quality}

From 636 samples derived from blood or cell line, 622 (97.80 \%) passed quality control based on recommended best practices for Axiom arrays by Affymetrix [55]. Several samples were excluded from subsequent analyses; one sample failed to scan, eight had Dish $\mathrm{QC}<0.82$ due to a sample processing issue, and five had stage one genotype call rates $<97 \%$. In addition, seven negative controls were processed across the genotyping plates, and all exhibited low separation from the background signal as expected. In passing samples, 582,143 (90.08 \%) of the markers on the array passed quality control, using Affymetrix recommended best practices. With default settings of Affymetrix SNPolisher classifications, we removed: 48,083 markers classified as "Other"; 7014 classified as "CallRateBelowThreshold"; 1765 classified as
"Off-target variant (OTV)"; 181 classified as "Hemizygous"; and 7061 classified as "MonoHighResolution" that also had previously failed Affymetrix's internal validation process (see Table 3).

The average genotype reproducibility in 25 replicate pairs across all passing SNPs was $99.94 \%$. Of the passing markers, 40,745 (7.00 \%) were monomorphic, 17,099 (2.94\%) had minor allele frequency (MAF) greater than 0 and less than $0.01 ; 125,359$ (21.53 \%) had MAF between 0.01 and $0.05 ; 398,940(68.53 \%)$ had MAF greater than or equal to 0.05. For 137 HapMap samples [56] (45 JPT, 32 CEU, 60 YRI) and 226,511 passing markers that overlapped with HapMap2 release 24 (marker call rate $\geq 95 \%$ in HapMap2), the average genotype concordance was $99.76 \%$.

\section{Smokescreen application to nicotine metabolism analysis in multiple populations}

Unrelated African American $(N=52)$, Asian American $(N=55)$, and European American ancestry individuals $(N=239)$ from three existing laboratory-based nicotine metabolism studies were selected for genotyping on the Smokescreen array [57]. For all samples, the NMR is defined as the trans-3'-hydroxycotinine to cotinine ratio. In these three studies, biospecimens were collected, and creatinine-adjusted cotinine and trans-3'-hydroxycotinine levels were estimated via mass spectrometry using an established method [57].

In the Pharmacokinetics in Twins ("PKTWIN") [58] study, participants were recruited from the Northern California Twin Registry in a multiple stage protocol to coordinate ascertainment of twins to investigate heritability and genetic components of nicotine metabolism. Participants consented to a 30-min venous administration of nicotine and cotinine, followed by an 8-hour hospital stay for blood and urine biosample collection. In the Pharmacogenetic 
Table 3 SNP classifications and recommendations using 622 passing samples derived from blood or cell line

\begin{tabular}{|c|c|c|c|c|c|}
\hline $\begin{array}{l}\text { SNPolisher } \\
\text { ConversionType }\end{array}$ & $\begin{array}{l}\text { Previously validated } \\
\text { markers }\end{array}$ & $\begin{array}{l}\text { Previously failed-validation } \\
\text { markers }\end{array}$ & $\begin{array}{l}\text { De novo } \\
\text { markers }\end{array}$ & Total SNPS & $\begin{array}{l}\text { Recommendation } \\
\text { to keep for analysis }\end{array}$ \\
\hline PolyHighResolution & $441120(68.26 \%)$ & $17595(2.72 \%)$ & $7547(1.17 \%)$ & $\begin{array}{l}466262 \\
(72.15 \%)\end{array}$ & yes \\
\hline NoMinorHom & 62639 (9.69 \%) & $6360(0.98 \%)$ & $6173(0.96 \%)$ & $\begin{array}{l}75172 \\
(11.63 \%)\end{array}$ & yes \\
\hline MonoHighResolution & 12095 (1.87 \%) & 7061 (1.09 \%) & $28614(4.43 \%)$ & $47770(7.39 \%)$ & $\begin{array}{l}\text { yes if previously } \\
\text { validated or de novo }\end{array}$ \\
\hline Other & $6031(0.93 \%)$ & 14515 (2.24 \%) & $27537(4.26 \%)$ & 48083 (7.44 \%) & no \\
\hline CallRateBelowThreshold & 3078 (0.48 \%) & 2856 (0.42 \%) & 1080 (0.17 \%) & 7014 (1.09 \%) & no \\
\hline OTV & $370(0.06 \%)$ & $535(0.08 \%)$ & $860(0.13 \%)$ & 1765 (0.27 \%) & $\begin{array}{l}\text { yes if off-target variant } \\
\text { genotyped }\end{array}$ \\
\hline Hemizygous & $180(0.03 \%)$ & $1(<0.01 \%)$ & $0(0.00 \%)$ & $181(0.03 \%)$ & yes if visually inspected \\
\hline TOTAL & $525513(81.32 \%)$ & 48923 (7.57 \%) & 71811 (11.11\%) & 646247 & \\
\hline
\end{tabular}

'Previously validated' or 'Previously failed-validation' are markers tested by the manufacturer. 'De novo' are markers on the array but not validated by the manufacturer. 'PolyHighResolution' and 'NoMinorHom' are markers with good cluster resolution. 'MonoHighResolution' indicates that fewer than two examples of the minor allele was present. 'CallRateBelowThreshold' indicated that the SNP call rate is below the threshold while other properties are above the threshold. 'Other' are markers where one or more cluster properties falls below its threshold

Study of Nicotine Metabolism (“588”) [57], recruiting by smoking status and gender of European, African, and Asian Americans was performed through multi-media advertisements for a nicotine and cotinine metabolism study. Participants consented to morning oral administration of nicotine, and either labeled (smokers) or unlabeled (nonsmokers) cotinine. The following biospecimens were collected: saliva up to $60 \mathrm{~h}$ after dosing; blood up to $480 \mathrm{~min}$; urine up to $8 \mathrm{~h}$. In the Smoking in Families (SMOFAM) study [59], individuals from 61 pedigrees with at least three ever-smokers individuals per pedigree originally recruited to assess the relations among genetic factors, environmental factors, and tobacco use, consented to oral administration of a fixed dose of nicotine and cotinine at home monitored by a nurse, followed by salivary sample collection at multiple time points as well as a blood sample for DNA analysis [57].

Three hundred fifteen samples passed quality control with complete data and were included in the analyses (from 49 African, 51 Asian, and 215 European American participants). Multiple factors including population structure principal components, age, sex, BMI, and smoking status were incorporated to model NMR in a genome-wide, multi-ethnic meta-analysis. Adequate Type I error control was observed with genome-wide significant results at CYP2A6 (min $p=9.10 \mathrm{E}-15)$. The top SNP accounts for $12 \%, 27 \%$, and $19 \%$ of the NMR variation in European Americans, African Americans, and Asian Americans, respectively. The minimum $p$ value in the nearby $C Y P 2 B 6$ region was $1.85 \mathrm{E}-5$ with association patterns differing by ancestry (Fig. 2). Association patterns with the NMR remained intact for $C Y P 2 B 6$ in an analysis adjusting for $C Y P 2 A 6$ 's top SNP; other $C Y P 2 A 6$ SNPs showed evidence of independent association after this adjustment $(\min p=1.71 \mathrm{E}-7)$.

\section{Discussion}

Until recently, manufacturing a genotyping array, especially with primarily custom-content, was prohibitively expensive. The Smokescreen array represents the next generation of targeted arrays, providing both baseline genome-wide content, and enhanced coverage of important regions and pathways specific to a phenotype or group of related phenotypes. The popularity of these similarly-sized arrays can be seen in the Psychiatric Genetics Consortium Infinium PsychArray [60] with 571,054 SNPs (271,406 genome-wide tag SNPs, 276,701 exonic markers, and 50,000 markers associated with common psychiatric disorders) and the OncoArray Consortium OncoArray-500 K [61] with 499,170 SNPs (275,691 genome-wide tag SNPs and 223,479 cancer specific SNPs). These three arrays offer a core set of markers that provide similar imputation-based genomewide coverage. Used a procedure similar to Nelson et al. [62], we estimated that the Smokescreen design covers $66 \%, 82 \%$, and $91 \%$ of common variants $(\mathrm{MAF} \geq 0.05)$ in YRI, ASN, and EUR, respectively. The Smokescreen array includes extensive custom content (350,209 markers), focusing on enhancing coverage of gene regions and pathways related to addiction (dependence, drug metabolism, and treatment response), and attributable disease (proliferative, psychiatric and pulmonary outcomes) in multiple populations. Consortia developed arrays have similar goals: to provide content of value to researchers studying related phenotypes and to provide data for large meta-analyses and replication studies.

Genome-wide arrays, such as Smokescreen, allow for imputation of common and rare genotypes using haplotype reference panels. Recent haplotype projects (e.g., the Haplotype Reference Consortium, which combines multiple cohorts including the $1000 \mathrm{Ge}$ nomes Project) allow for more accurate imputation of 


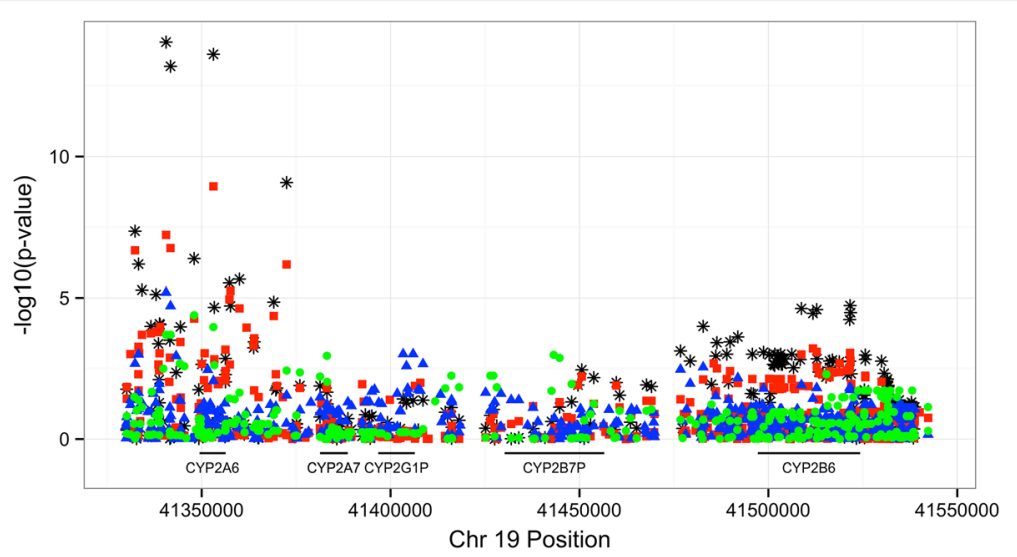

Fig. 2 CYP2A6 - CYP2B6 regional association with the nicotine metabolism ratio. Blue triangle: African-American. Green circle: Asian-American. Red square: European-American. Black star: Meta-analysis

less common and rare variants [63, 64]. The ability to genotype large numbers of study samples and impute more variants accurately, enables powerful meta-GWAS studies [65]. Additionally, genome-wide imputation allows for discovery of associations in regions not directly enriched for in the array design. This is important as meta-analysis consortia and multiple methods [51] identify additional addiction-related regions.

There are some limitations in the estimation of Smokescreen array performance. It should be noted that the coverage is an estimate based on the design of the array. The realized coverage is not yet computable as the validation of markers is ongoing. Approximately $12 \%$ of SNPs on the array are too rare to be observed with the current sample size. Additional samples will improve genotype clustering of rare SNPs or SNPs with low quality metrics. Some pilot samples derived from saliva were genotyped, but the sample size was not sufficient for estimating performances on these samples separately. In general, we recommend saliva samples to be collected using kits with preservatives. Laboratories familiar with Axiom Arrays should assess the quality of salivary samples with suspect collection or storage method prior to genotyping.

Ascertainment biases in the markers selected on the array or the reference population used in imputation may lead to lower coverage, as commonly observed in African ancestry populations. However, increasing coverage on the array (as we did with the African (YRI) booster panel) and diverse reference panels for imputation, helps mitigate this issue. Another limitation of these approaches over sequencing, is the identification of de novo mutations. These mutations, however, account for a small fraction of both rare and common neurodevelopmental diseases and require pedigrees for analysis [66].

We envision the Smokescreen array driving translational research by facilitating the development of algorithms, derived from multiple genetic and clinical factors for risk prediction and treatment approach assignments. Previously, genome-wide allelotyping analyses of smoking cessation trials revealed associations of common variants with prospective abstinence [67]. This research lead to the design of a clinical trial analysis model incorporating a "quit-success" genetic score, which retrospectively predicted abstinence in a randomized trial stratifying smokers by nicotine replacement therapy dose and dependence [53]. This model used both genetic ("quit-success" score) and clinical (FTCD score) information. We re-envision this model based on a Smokescreen analysis platform that incorporates individual level genotype data, additional clinical factors, and the multi-stage process of validation and utility assessment in large sample sizes derived from meta-analysis of multiple trials [68]. For example, genotyping samples with multiple addiction-related phenotypes will permit genome-wide correlation [69-71] and estimation of the extent of shared variance and polygenicity among dependence, attributable disease, and treatment response; the proportion of shared variance among dependencies using genome-wide correlation is substantial [6].

The addiction-related gene content of Smokescreen is designed to be helpful in pharmacogenetic analyses of current or future addiction gene targets. In an analysis of the Psychiatric Genetic Consortium schizophrenia findings [72], 40 of the 341 protein-coding genes linked to GWAS hits were identified as targets of existing drugs or drugs undergoing Phase III trials [73]. Lencz and Malhotra conclude that six proteincoding genes (CACNA1C, CACNB2, CACNA1I, DRD2, GRIN2A, HCN1) are of greatest neuropsychiatric and genetic interest. Three of these six genes (DRD2, CACNA1I, GRIN2A) are included in Smokescreen's group of addiction-related genes; overall, 10 of the 40 target genes are included in Smokescreen's gene list 
with increased coverage, suggesting Smokescreen's usefulness for addiction-related drug development studies. Coverage of the nicotine metabolizing enzyme genes CYP2A6 and CYP2B6, the opportunity to incorporate NMR measures in larger studies, and novel analytical methods may improve nicotine metabolism models, which currently predict between 50 and $70 \%$ of variance in European ancestry populations [39].

\section{Conclusions}

The Smokescreen array achieves robust genome-wide coverage of common variants, and exceptional coverage of 1014 genes relevant to addiction and known nicotine metabolism and smoking behavior regions in African (YRI), Asian, and European populations. These, paired with content from recent findings and related work in pharmacogenomics, comorbidities, and attributable diseases create a comprehensive genotyping array for addiction research. Analytical validity and utility were demonstrated through pilot genotyping of HapMap and study samples. For HapMap samples, the genotype concordance for overlapping content was $>99 \%$. Based on samples from nicotine metabolism laboratory studies, we identified variants at genome-wide significance in a region known to be highly influential on nicotine metabolism, serving as a positive confirmation of the array's design. These attributes enable researchers to perform genome-wide, candidate gene, and pathway-based association analyses on various addictions, including those related to smoking and tobacco use.

\section{Methods}

\section{Array design objectives}

The array design was driven by the need for a common panel of markers for both hypothesis-driven and genomewide studies of addiction. This project was funded by the Small Business Innovative Research (SBIR) program with the National Institute on Drug Abuse (NIDA). Specific requirements included capturing prioritized markers identified by NIDA; selecting focused content for a wide variety of research purposes and multiple ethnicities; selecting a platform for accurate and reproducible data across studies, and providing a screening tool for development of risk assessments and personalized approaches to addiction treatment.

The ability to customize the array was important to cover common and rare variation across populations genome-wide, with enhanced coverage in genes and regions related to addiction. Manufacturing reproducibility was also critical for translational and clinical usecases that may be developed based on the array. Given these objectives, Affymetrix Axiom was selected as a platform for the array.

\section{Content targeted for coverage}

Content was selected for inclusion on the array in a modular fashion and then prioritized to create the final specifications for manufacturing. The content was compiled from multiple sources, including pathway and functional databases, NIDA, NIH-funded investigators, prior genotyping and sequencing projects, Affymetrix, and scientific publications.

\section{Genome-wide association markers}

A catalog panel was provided by Affymetrix to provide baseline genome-wide coverage across AFR, ASN, and EUR ancestry groups for discovery as well as metaanalysis across studies. 246,038 of the catalog markers overlap with the Affymetrix Axiom Biobank array. We included an additional panel of 50,000 markers provided by Affymetrix to increase genome-wide coverage in African ancestry populations, thereby maximizing genotype imputation efficiency to a larger variant set $[74,75]$.

\section{Addiction-related genes}

One thousand fourteen genes were identified as related to addiction through expert nomination, and recent bioinformatics projects and knowledge-bases. Table 4 presents the source, annotation, and gene count. Sources include the NIDA Genetics Consortium [76, 77], Gene Ontology [78], QIAGEN's Ingenuity Pathway Analysis [79], and Pharmacogenetics of Nicotine Addiction Treatment [31, 37].

\section{Fine mapping of smoking-related loci}

We aimed for the densest coverage of any genotyping array for the chr15q25.1 nicotinic acetylcholine receptor (nAChR) gene cluster (CHRNA5, CHRNA3 and CHRNB4), and the chr19q13.2 nicotine metabolizing enzyme genes (CYP2A6 and CYP2B6). The CYP2A6 gene plays a major role in the nicotine metabolism pathway $[80,81]$ while genes encoding for CYP isozymes, such as the CYP2B6 gene, may play a smaller role in influencing nicotine metabolism [82, 83]. An individual's nicotine metabolism affects the level of circulating and sequestered nicotine and thus, nicotine intake [40, 84]. Nicotine binds to nAChRs, triggering neurotransmitter release and leads over time to nicotine dependence. nAChR activity, and thus nicotine dependence, is regulated by the cholinergic genes on chromosomes 8p11.21, 15q25.1 and 20q13.33 [16, 17, 21, 85-88].

\section{Filtering and tagging of selected markers}

Each content category was submitted to Affymetrix as a list of markers or genomic regions. Affymetrix used proprietary software and a Axiom-validated marker database to select the best-performing markers (one or more probesets per marker) that covered the targeted content, either through direct inclusion or through efficient pairwise tagging. Multiple probesets were selected for markers that are either 
Table 4 Smokescreen addiction genes: source, categories, and counts*

\begin{tabular}{|c|c|c|}
\hline Source & Category & Genes \\
\hline Gene Ontology & $\begin{array}{l}\text { dopamine_receptor__ } \\
\text { binding }\end{array}$ & 8 \\
\hline Gene Ontology & dopamine_binding & 9 \\
\hline Gene Ontology & serotonin_uptake & 4 \\
\hline Gene Ontology & $\begin{array}{l}\text { serotonin_metabolic_ } \\
\text { process }\end{array}$ & 8 \\
\hline Gene Ontology & serotonin_transport & 12 \\
\hline Gene Ontology & response_to_nicotine & 31 \\
\hline Gene Ontology & dopamine_secretion & 15 \\
\hline Gene Ontology & dopamine_uptake & 8 \\
\hline Gene Ontology & $\begin{array}{l}\text { dopamine_receptor_- } \\
\text { signaling_pathway }\end{array}$ & 30 \\
\hline Gene Ontology & dopamine_transport & 23 \\
\hline Gene Ontology & serotonin_secretion & 8 \\
\hline Gene Ontology & $\begin{array}{l}\text { dopamine_metabolic_ } \\
\text { process }\end{array}$ & 26 \\
\hline Gene Ontology & $\begin{array}{l}\text { regulation_of_dopamine_ } \\
\text { secretion }\end{array}$ & 15 \\
\hline IPA & $\begin{array}{l}\text { cigarette_habituation_ } \\
\text { susceptibility_syndrome }\end{array}$ & 6 \\
\hline IPA & nicotine & 14 \\
\hline IPA & $\begin{array}{l}\text { susceptibility_to_drug_ } \\
\text { addiction }\end{array}$ & 1 \\
\hline IPA & addiction_behavior & 24 \\
\hline IPA & tobacco & 27 \\
\hline IPA & addiction & 131 \\
\hline IPA & withdrawal & 11 \\
\hline IPA & smoking & 12 \\
\hline IPA & naltrexone & 4 \\
\hline IPA & clonidine & 7 \\
\hline IPA & nortripyline & 3 \\
\hline IPA & varenicline & 3 \\
\hline IPA & nicotine & 19 \\
\hline IPA & bupropion & 3 \\
\hline NIDA Genetics Consortium & The Nicotine System & 20 \\
\hline NIDA Genetics Consortium & The Dopamine System & 10 \\
\hline NIDA Genetics Consortium & Mouse QTL & 423 \\
\hline NIDA Genetics Consortium & The Alcohol System & 32 \\
\hline NIDA Genetics Consortium & The Cholinergic System & 9 \\
\hline NIDA Genetics Consortium & The Adrenergic System & 16 \\
\hline NIDA Genetics Consortium & Tyrosine & 3 \\
\hline NIDA Genetics Consortium & Other & 263 \\
\hline NIDA Genetics Consortium & The GABA System & 31 \\
\hline NIDA Genetics Consortium & $\begin{array}{l}\text { Neurotransmitter } \\
\text { Transporters }\end{array}$ & 13 \\
\hline
\end{tabular}

NIDA Genetics Consortium
Table 4 Smokescreen addiction genes: source, categories, and counts* (Continued)

\begin{tabular}{lll}
\hline & $\begin{array}{l}\text { The Nicotine Metabolism } \\
\text { System }\end{array}$ & \\
NIDA Genetics Consortium & The Serotonergic System & 20 \\
NIDA Genetics Consortium & The Endocannabinoid System & 2 \\
NIDA Genetics Consortium & Dopamine Synthesis & 2 \\
NIDA Genetics Consortium & The Glutamatergic System & 42 \\
NIDA Genetics Consortium & The Opioid System & 12 \\
PNAT & & 134 \\
\hline${ }^{*}$ Genes in categories may overlap &
\end{tabular}

non-validated or deemed high priority (e.g., markers with known associations with addiction, smoking behavior, or nicotine metabolism), in order to minimize genotyping failures of these markers.

Using genotype data from the 1000 Genomes Project (Phase 1, March 2012 release), in African (YRI), East Asian $(\mathrm{CHB}+\mathrm{CHS}+\mathrm{JPT})$ and European (CEU + FIN + $\mathrm{GBR}+\mathrm{IBS}+\mathrm{TSI}$ ) populations, tagging the 1014 addictionrelated genes $( \pm 20 \mathrm{~kb})$ was performed in three rounds: (1) all markers with an MAF $\geq 0.05$ were tagged at $r^{2} \geq 0.9$ using Axiom-validated markers only; (2) remaining untagged markers were tagged at $r^{2} \geq 0.8$ using Axiomvalidated markers only; (3) any remaining untagged markers were tagged at $r^{2} \geq 0.9$ using non-validated markers.

Both the range encompassing the entirety of CYP2A6 and CYP2B6 genes $( \pm 20 \mathrm{~kb})$ and the range defined by the largest linkage disequilibrium (LD) block encompassing the nAChR gene cluster were defined. All known variants were selected from multiple databases, including 1000 Genomes Project Phase 1, HapMap, and the NHLBI GO Exome Sequencing Project [89].

\section{Imputed coverage estimation}

Imputation coverage was estimated for the Smokescreen array using an approach similar to that described by Nelson et al. [62]. 1000 Genomes Project Phase 1 data (March 2012 release) were extracted for the Smokescreen content as an imputation inference set. All 1000 Genomes Project Phase 1 SNPs were used as the reference set. For each population (EUR, ASN, YRI), groups of 10 samples were created. For each group, the samples were kept in the inference set and excluded from the reference set, and imputed separately from other groups. Samples with known or cryptic relatedness with other samples in the 1000 Genomes Project were excluded both from the inference set groups and reference sets. Beagle version v4.0 release 1230 was used for phasing and imputation of chromosomes 1 to 22 with default settings [90]. Imputation was broken up by chromosome and results were then combined for all SNPs and groups of samples. For each SNP, we computed the correlation 
Table 5 Smokescreen pilot genotyping sample characteristics - blood or cell line

\begin{tabular}{|c|c|c|c|c|c|}
\hline Study & Description & Samples & Individuals & DNA Source & \%Male \\
\hline Hapmap and 1000 Genomes & $\begin{array}{l}\text { CEPH/Utah; Yoruba in Ibadan, Nigeria; } \\
\text { Japanese in Tokyo, Japan samples }\end{array}$ & 188 & 188 & cell line & $50.00 \%$ \\
\hline PKTWIN, "588”, SMOFAM & Nicotine metabolism laboratory studies & 343 & 326 & blood & $45.71 \%$ \\
\hline Total Exposure Study & Cross sectional study of tobacco exposures & 33 & 32 & blood & $56.25 \%$ \\
\hline MA fMRI, Modafinil, ASCC2, ASCC Neural Systems & Genetics and brain structure in smokers & 35 & 34 & blood & 58.829 \\
\hline COGEND & Report of genetic results in smokers & 31 & 30 & blood & 33.339 \\
\hline Positive lab controls & & 6 & 1 & cell line & \\
\hline
\end{tabular}

between the imputed dosages and measured genotypes from the 1000 Genomes Project (obsRSQ). The obsRSQ were then summarized overall (genome-wide), for the addiction-genes and the fine-mapping regions for each population.

\section{Smokescreen genotyping pilot}

DNA samples from 188 Hapmap and 1000 Genomes Project participants from CEPH/Utah, Yoruba in Ibadan, Nigeria, and Japanese in Tokyo, Japan populations were obtained from Coriell Institute for Medical Research (Camden, NJ); 442 study samples from NIDA-funded Investigators, and six positive controls provided by the genotyping lab, were obtained for the genotyping pilot (see Table 5).

\section{Ethics approval and consent to participate}

All individuals provided written informed consent. The Collaborative Genetic Study of Nicotine Dependence (COGEND) study was approved by the Washington University Institutional Review Board. The Early Methamphetamine Abstinence: fMRI and Cognition (MA fMRI); Neural Systems, Inhibitory Control, and Methamphetamine Dependence (Modafinil); Neural Systems and Risk for Adolescent Smoking (ASCC2); and Neural Correlates of Cognition, Craving and Reward Delay in Adolescent Smokers (ASCC Neural Systems) studies were approved by the University of California, Los Angeles Institutional Review Board. The Total Exposure Study (TES) was approved by the SRI International Human Subjects Committee. The Pharmacokinetics in Twins (PKTWIN), Pharmacogenetic Study of Nicotine Metabolism (588), and Smoking in Families (SMOFAM) studies were approved by the committee on Human Research at the University of California San Francisco and the Human Subjects Committee at SRI International.

A total of 636 purified DNA samples (including 27 replicates) derived from blood or cell line were processed on the Smokescreen Genotyping Array at RUCDR Infinite Biologics (Piscataway, NJ) according to manufacturer instructions. Raw data consisted of one CEL file per sample (except for one sample that failed to scan).
Following best practices guidelines set forth by the manufacturer [55], the Affymetrix Power Tools (APT) v1.17.0 software was used to process raw data. Dish QC (DQC) values were generated and used to remove samples with $\mathrm{DQC}<0.82$. As part of stage 1 genotyping, 20,000 probesets previously validated by the manufacturer were used to cluster genotypes and remove samples with stage 1 call rate $<97 \%$. Plate pass rate and average stage 1 call rate per plate were calculated and reviewed to determine if any plates should be excluded from further analysis. Remaining samples were genotyped for all probesets (stage 2 genotyping), using APT. The Affymetrix SNPolisher v1.5.2 software was used to classify probeset quality and determine the best probeset for each marker. Markers whose best probeset classified as "Other", "CallRateBelowThreshold", "Off-target variant (OTV)", "Hemizygous", or "MonoHighResolution" (or had previously failed Affymetrix' internal validation process) were removed. A smaller number of DNA samples derived from saliva were also genotyped and clustered separately but are excluded here. Prior to statistical analysis, samples with sex discrepancies (reported versus expected), unexpected relatedness (half-sib or greater), and replicate samples with the lowest call rate were excluded. All statistical analyses were performed in [R] [91].

\section{Additional file}

Additional file 1: Smokescreen addiction regions and estimated imputed coverage. This file contains the addiction-related gene regions (chr 1-22) represented on the Smokescreen array and the estimated imputation-based coverage for each region in European (EUR), Asian (ASN), and African (YRI) populations. (XLSX $338 \mathrm{~kb}$ )

\footnotetext{
Abbreviations

ADME: Absorption, distribution, metabolism, and excretion; AFR: African; AIM: Ancestry informative marker; APT: Affymetrix Power Tools; ASN: Asian; CEU: Utah Residents (CEPH) with Northern and Western European Ancestry; CHB: Han Chinese in Beijing, China; CHS: Southern Han Chinese; CPD: Cigarettes per day; DQC: Dish QC; EUR: European; FIN: Finnish in Finland; FTCD: Fagerström Test for Cigarette Dependence; GBR: British in England and Scotland; GWAS: Genome-wide association study; IBS: Iberian Population in Spain; IPA: Ingenuity Pathway Analysis; JPT: Japanese in Tokyo, Japan; LD: Linkage disequilibrium; MAF: Minor allele frequency; nAChR: Nicotinic acetylcholine receptor; NIDA: National Institute on Drug
} 
Abuse; NMR: Nicotine Metabolite Ratio; NRT: Nicotine Replacement Therapy OTV: Off-target variant; PNAT: Pharmacogenetics of Nicotine Addiction Treatment; SBIR: Small Business Innovative Research; SNP: Single nucleotide polymorphism; TSI: Toscani in Italia; YRI: Yoruba in Ibadan, Nigeria.

\section{Competing interests}

BioRealm LLC intends to commercialize the Smokescreen array.

\section{Authors' contributions}

JWB, CKE co-first authors, project leaders, developed the array. JWB, CKE, $A W B, D V C$ contributed to the design of the array. JWB, CKE, CIP ran the analysis. JWB, CKE, CIP, DVC, AWB wrote the paper. All authors read and approved the final manuscript.

\section{Acknowledgements}

This project has been funded in whole or in part with Federal funds from the National Institute on Drug Abuse, National Institutes of Health, Department of Health and Human Services, under Contract Nos. HHSN271201200005C and HHSN271201300004C, and Grant No. DA033813. Computing was supported by an AWS in Education Research Grant award. We would like to acknowledge the NIDA Genetics Consortium, the Psychiatric Genetics Consortium, and other investigators for suggesting content; the Affymetrix bioinformatics team and RUCDR Infinite Biologics for working closely with us in developing the array, QC procedures, and sample processing; the Investigators contributing pilot samples; and the entire BioRealm team (http://biorealmresearch.com).

\section{Received: 4 September 2015 Accepted: 17 February 2016}

\section{Published online: 27 February 2016}

\section{References}

1. The Science of Drug Abuse and Addiction: The Basics | National Institute on Drug Abuse (NIDA). http://www.drugabuse.gov/publications/media-guide/ science-drug-abuse-addiction-basics. Accessed 20 Nov 2015.

2. American Psychiatric Association. Diagnostic and Statistical Manual of Mental Disorders: DSM-5. 5th ed. Arlington, VA: American Psychiatric Publishing; 2013.

3. Agrawal A, Verweij KJH, Gillespie NA, Heath AC, Lessov-Schlaggar CN, Martin $N G$, et al. The genetics of addiction - a translational perspective. Transl Psychiatry. 2012;2:e193.

4. Ducci F, Goldman D. The genetic basis of addictive disorders. Psychiatr Clin North Am. 2012;35:495-519.

5. Vrieze SI, McGue M, Miller MB, Hicks BM, lacono WG. Three mutually informative ways to understand the genetic relationships among behavioral disinhibition, alcohol use, drug use, nicotine use/dependence, and their cooccurrence: twin biometry, GCTA, and genome-wide scoring. Behav Genet. 2013:43:97-107.

6. Palmer RHC, Brick L, Nugent NR, Bidwell LC, McGeary JE, Knopik VS, et al. Examining the role of common genetic variants on alcohol, tobacco, cannabis and illicit drug dependence: genetics of vulnerability to drug dependence. Addiction. 2015;110:530-7.

7. Kendler KS, Myers J, Prescott CA. Specificity of genetic and environmental risk factors for symptoms of cannabis, cocaine, alcohol, caffeine, and nicotine dependence. Arch Gen Psychiatry. 2007;64:1313-20.

8. United States Surgeon General. The Health Consequences of Smoking - 50 Years of progress: A Report of the Surgeon General. PsycEXTRA Dataset. 2014

9. Heatherton TF, Kozlowski LT, Frecker RC, Fagerström KO. The Fagerström Test for Nicotine Dependence: a revision of the Fagerström Tolerance Questionnaire. Br J Addict. 1991;86:1119-27.

10. Fagerström K. Determinants of tobacco use and renaming the FTND to the Fagerstrom Test for Cigarette Dependence. Nicotine Tob Res. 2012;14:75-8.

11. Sullivan PF, Kendler KS. The genetic epidemiology of smoking. Nicotine Tob Res. 1999;1 Suppl 2:S51-7. discussion S69-70.

12. Bares CB, Kendler KS, Maes HH. Developmental Changes in Genetic and Shared Environmental Contributions to Smoking Initiation and Subsequent Smoking Quantity in Adolescence and Young Adulthood. Twin Res Hum Genet. 2015:18(5):497-506. doi:10.1017/thg.2015.48.

13. Pergadia ML, Agrawal A, Loukola A, Montgomery GW, Broms U, Saccone SF, et al. Genetic linkage findings for DSM-IV nicotine withdrawal in two populations. Am J Med Genet B Neuropsychiatr Genet. 2009;150B:950-9.
14. Han S, Gelernter J, Luo X, Yang B-Z. Meta-analysis of 15 genome-wide linkage scans of smoking behavior. Biol Psychiatry. 2010;67:12-9.

15. Berrettini W, Yuan X, Tozzi F, Song K, Francks C, Chilcoat H, et al. Alpha-5/ alpha-3 nicotinic receptor subunit alleles increase risk for heavy smoking. Mol Psychiatry. 2008;13:368-73.

16. Thorgeirsson TE, Geller F, Sulem P, Rafnar T, Wiste A, Magnusson KP, et al. A variant associated with nicotine dependence, lung cancer and peripheral arterial disease. Nature. 2008:452:638-42.

17. Thorgeirsson TE, Gudbjartsson DF, Surakka I, Vink JM, Amin N, Geller F, et al. Sequence variants at CHRNB3-CHRNA6 and CYP2A6 affect smoking behavior. Nat Genet. 2010;42:448-53.

18. Tobacco and Genetics Consortium. Genome-wide meta-analyses identify multiple loci associated with smoking behavior. Nat Genet. 2010;42:441-7.

19. David SP, Hamidovic A, Chen GK, Bergen AW, Wessel J, Kasberger JL, et al. Genome-wide meta-analyses of smoking behaviors in African Americans. Transl Psychiatry. 2012;2:e119.

20. Bloom AJ, Hartz SM, Baker TB, Chen L-S, Piper ME, Fox L, et al. Beyond cigarettes per day. A genome-wide association study of the biomarker carbon monoxide. Ann Am Thorac Soc. 2014;11:1003-10.

21. Rice JP, Hartz SM, Agrawal A, Almasy L, Bennett S, Breslau N, et al. Consortium, Geneva: CHRNB3 is more strongly associated with Fagerstrom test for cigarette dependence-based nicotine dependence than cigarettes per day: phenotype definition changes genome-wide association studies results. Addiction. 2012;107:2019-28.

22. Bierut LJ, Madden PAF, Breslau N, Johnson EO, Hatsukami D, Pomerleau OF et al. Novel genes identified in a high-density genome wide association study for nicotine dependence. Hum Mol Genet. 2007;16:24-35.

23. Thun MJ, Carter BD, Feskanich D, Freedman ND, Prentice R, Lopez AD, et al. 50 -year trends in smoking-related mortality in the United States. N Engl J Med. 2013:368:351-64

24. Centers for Disease Control and Prevention (CDC). Vital signs: current cigarette smoking among adults aged $>$ or $=18$ years - United States, 2009. MMWR Morb Mortal Wkly Rep. 2010;59:1135-40.

25. Centers for Disease Control and Prevention (CDC). Quitting smoking among adults-United States, 2001-2010. MMWR Morb Mortal Wkly Rep. 2011;60:1513-9.

26. Fiore MC, Bailey WC, Cohen SJ, Dorfman SF, Goldstein MG, Gritz ER, et al. Treating Tobacco Use and Dependence: A Clinical Practice Guideline. Rockville, MD: US Dept of Health and Human Services; 2000. AHRO publication No. 00-0032

27. Tobacco TCPGT et al. A clinical practice guideline for treating tobacco use and dependence: 2008 update: a US public health service report. Am J Prev Med. 2008;35:158-76.

28. Xian H, Scherrer JF, Madden PAF, Lyons MJ, Tsuang M, True WR, et al. The heritability of failed smoking cessation and nicotine withdrawal in twins who smoked and attempted to quit. Nicotine Tob Res. 2003;5:245-54.

29. Broms U, Silventoinen K, Madden PAF, Heath AC, Kaprio J. Genetic architecture of smoking behavior: a study of Finnish adult twins. Twin Res Hum Genet. 2006:9:64-72.

30. Mamoun M, Bergen AW, Shieh J, Wiggins A, Brody AL. Biomarkers of Response to Smoking Cessation Pharmacotherapies: Progress to Date. CNS Drugs. 2015;29:359-69.

31. Conti DV, Lee W, Li D, Liu J, Van Den Berg D, Thomas PD, et al. Pharmacogenetics of Nicotine A, Treatment, Consortium: Nicotinic acetylcholine receptor beta2 subunit gene implicated in a systems-based candidate gene study of smoking cessation. Hum Mol Genet. 2008;17:2834-48.

32. King DP, Paciga S, Pickering E, Benowitz NL, Bierut LJ, Conti DV, et al. Smoking cessation pharmacogenetics: analysis of varenicline and bupropion in placebocontrolled clinical trials. Neuropsychopharmacology. 2012;37:641-50.

33. Tyndale RF, Zhu AZX, George TP, Cinciripini P, Hawk Jr LW, Schnoll RA, et al. Lack of Associations of CHRNA5-A3-B4 Genetic Variants with Smoking Cessation Treatment Outcomes in Caucasian Smokers despite Associations with Baseline Smoking. PLoS One. 2015;10:e0128109.

34. Chen L-S, Baker TB, Piper ME, Breslau N, Cannon DS, Doheny KF, et al. Interplay of genetic risk factors (CHRNA5-CHRNA3-CHRNB4) and cessation treatments in smoking cessation success. Am J Psychiatry. 2012;169:735-42.

35. Baker TB, Weiss RB, Bolt D, von Niederhausern A, Fiore MC, Dunn DM, et al. Human neuronal acetylcholine receptor A5-A3-B4 haplotypes are associated with multiple nicotine dependence phenotypes. Nicotine Tob Res. 2009;11:785-96.

36. Munafo MR, Johnstone EC, Walther D, Uhl GR, Murphy MF, Aveyard P. CHRNA3 rs 1051730 Genotype and Short-term Smoking Cessation. Nicotine Tob Res. 2011;13(10):982-8. 
37. Bergen AW, Javitz HS, Krasnow R, Nishita D, Michel M, Conti DV, et al. Nicotinic acetylcholine receptor variation and response to smoking cessation therapies. Pharmacogenet Genomics. 2013;23:94-103.

38. Benowitz NL, Swan GE, Jacob 3rd P, Lessov-Schlaggar CN, Tyndale RF. CYP2A6 genotype and the metabolism and disposition kinetics of nicotine. Clin Pharmacol Ther. 2006:80:457-67.

39. Bloom J, Hinrichs AL, Wang JC, von Weymarn LB, Kharasch ED, Bierut LJ, et al. The contribution of common CYP2A6 alleles to variation in nicotine metabolism among European-Americans. Pharmacogenet Genomics. 2011; 21:403-16.

40. Tyndale RF, Sellers EM. Variable CYP2A6-mediated nicotine metabolism alters smoking behavior and risk. Drug Metab Dispos. 2001;29:548-52.

41. Bloom AJ, Harari O, Martinez M, Madden PA, Martin NG, Montgomery GW, et al. Use of a predictive model derived from in vivo endophenotype measurements to demonstrate associations with a complex locus, CYP2A6. Hum Mol Genet. 2012;21:3050-62.

42. Lerman C, Tyndale R, Patterson F, Wileyto EP, Shields PG, Pinto A, et al. Nicotine metabolite ratio predicts efficacy of transdermal nicotine for smoking cessation. Clin Pharmacol Ther. 2006;79:600-8.

43. Patterson F, Schnoll RA, Wileyto EP, Pinto A, Epstein LH, Shields PG, et al. Toward personalized therapy for smoking cessation: a randomized placebocontrolled trial of bupropion. Clin Pharmacol Ther. 2008;84:320-5.

44. Schnoll RA, Patterson F, Wileyto EP, Tyndale RF, Benowitz N, Lerman C. Nicotine metabolic rate predicts successful smoking cessation with transdermal nicotine: a validation study. Pharmacol Biochem Behav. 2009;92:6-11

45. Lerman C, Schnoll RA, Hawk Jr LW, Cinciripini P, George TP, Wileyto EP, et al. Use of the nicotine metabolite ratio as a genetically informed biomarker of response to nicotine patch or varenicline for smoking cessation: a randomised, double-blind placebo-controlled trial. Lancet Respir Med. 2015;3(2):131-8.

46. Ho MK, Mwenifumbo JC, Al Koudsi N, Okuyemi KS, Ahluwalia JS, Benowitz $\mathrm{NL}$, et al. Association of nicotine metabolite ratio and CYP2A6 genotype with smoking cessation treatment in African-American light smokers. Clin Pharmacol Ther. 2009;85:635-43.

47. Lerman C, Jepson C, Wileyto EP, Patterson F, Schnoll R, Mroziewicz M, et al. Genetic variation in nicotine metabolism predicts the efficacy of extendedduration transdermal nicotine therapy. Clin Pharmacol Ther. 2010;87:553-7.

48. Chen LS, Bloom AJ, Baker TB, Smith SS, Piper ME, Martinez M, et al. Pharmacotherapy effects on smoking cessation vary with nicotine metabolism gene (CYP2A6). Addiction. 2014;109:128-37.

49. 1000 Genomes Project Consortium, Abecasis GR, Auton A, Brooks LD, DePristo MA, Durbin RM, et al. An integrated map of genetic variation from 1,092 human genomes. Nature. 2012;491:56-65.

50. Bahcall O, Orli B. COGS project and design of the iCOGS array. Nat Genet. 2013;45(4):343. doi:10.1038/ng.2592.

51. Sullivan PF. The psychiatric GWAS consortium: big science comes to psychiatry. Neuron. 2010;68:182-6.

52. Allen N, Sudlow C, Downey P, Peakman T, Danesh J, Elliott P, et al. UK Biobank: Current status and what it means for epidemiology. Health Policy Technol. 2012;1:123-6.

53. Rose JE, Behm FM, Drgon T, Johnson C, Uhl GR. Personalized smoking cessation: interactions between nicotine dose, dependence and quitsuccess genotype score. Mol Med. 2010;16:247-53.

54. A Catalog of Published Genome-Wide Association Studies www.genome. gov/gwastudies. Accessed 20 Nov 2015.

55. Axiom Genotyping Solution Data Analysis Guide. Affymetrix Inc., Santa Clara, CA, 2015. http://media.affymetrix.com/support/downloads/manuals/axiom_ genotyping_solution_analysis_guide.pdf. Accessed 20 Nov 2015.

56. International HapMap Project. http://hapmap.ncbi.nlm.nih.gov/. Accessed 1 Sept 2013.

57. Dempsey D, Tutka P, Jacob 3rd P, Allen F, Schoedel K, Tyndale RF, et al. Nicotine metabolite ratio as an index of cytochrome P450 2 A6 metabolic activity. Clin Pharmacol Ther. 2004;76:64-72.

58. Swan GE, Benowitz NL, Jacob 3rd P, Lessov CN, Tyndale RF, Wilhelmsen K, et al. Pharmacogenetics of nicotine metabolism in twins: methods and procedures. Twin Res. 2004;7:435-48.

59. Swan GE, Hudmon KS, Jack LM, Hemberger K, Carmelli D, Khroyan TV, et al. Environmental and genetic determinants of tobacco use: methodology for a multidisciplinary, longitudinal family-based investigation. Cancer Epidemiol Biomarkers Prev. 2003;12:994-1005.
60. Infinium PsychArray BeadChips. San Diego, CA: Illumina, Inc.; 2014. http:// www.llumina.com/products/psycharray.html. Accessed 20 Nov 2015.

61. Infinium OncoArray-550 K BeadChips. San Diego, CA: Illumina, Inc.; 2014. https://support.illumina.com/array/array_kits/infinium-oncoarray-500k-v1-0beadchip-kit.html. Accessed 20 Nov 2015.

62. Nelson SC, Doheny KF, Pugh EW, Romm JM, Ling H, Laurie CA, et al. Imputation-based genomic coverage assessments of current human genotyping arrays. G3. 2013;3:1795-807.

63. Lee $\mathrm{S}$, Abecasis GR, Boehnke M, Lin X. Rare-variant association analysis: study designs and statistical tests. Am J Hum Genet. 2014;95:5-23.

64. Haplotype Reference Consortium. http://www.haplotype-referenceconsortium.org. Accessed 20 Nov 2015.

65. Yang J, Bakshi A, Zhu Z, Hemani G, Vinkhuyzen AAE, Lee SH, et al. Genetic variance estimation with imputed variants finds negligible missing heritability for human height and body mass index. Nat Genet. 2015;47(10): 1114-20.

66. Ku CS, Polychronakos C, Tan EK, Naidoo N, Pawitan Y, Roukos DH, et al. A new paradigm emerges from the study of de novo mutations in the context of neurodevelopmental disease. Mol Psychiatry. 2013;18:141-53.

67. Uhl GR, Liu Q-R, Drgon T, Johnson C, Walther D, Rose JE, et al. Molecular genetics of successful smoking cessation: convergent genome-wide association study results. Arch Gen Psychiatry. 2008;65:683-93.

68. Committee on the Review of Omics-Based Tests for Predicting Patient Outcomes in Clinical Trials, Board on Health Care Services, Board on Health Sciences Policy, Institute of Medicine. Evolution of Translational Omics: Lessons Learned and the Path Forward. Washington (DC): National Academies Press (US); 2014.

69. Yang J, Lee SH, Goddard ME, Visscher PM. Genome-wide complex trait analysis (GCTA): methods, data analyses, and interpretations. Methods Mol Biol. 2013;1019:215-36.

70. Yang J, Lee SH, Goddard ME, Visscher PM. GCTA: a tool for genome-wide complex trait analysis. Am J Hum Genet. 2011;88:76-82.

71. Bulik-Sullivan BK, Loh P-R, Finucane HK, Ripke S, Yang J, Schizophrenia Working Group of the Psychiatric Genomics Consortium, et al. LD Score regression distinguishes confounding from polygenicity in genome-wide association studies. Nat Genet. 2015;47:291-5.

72. Schizophrenia Working Group of the Psychiatric Genomics Consortium. Biological insights from 108 schizophrenia-associated genetic loci. Nature. 2014;511:421-7.

73. Lencz T, Malhotra AK. Targeting the schizophrenia genome: a fast track strategy from GWAS to clinic. Mol Psychiatry. 2015;20:820-6.

74. Hoffmann TJ, Zhan Y, Kvale MN, Hesselson SE, Gollub J, Iribarren C, et al. Design and coverage of high throughput genotyping arrays optimized for individuals of East Asian, African American, and Latino race/ethnicity using imputation and a novel hybrid SNP selection algorithm. Genomics. 2011;98: 422-30.

75. Hoffmann TJ, Kvale MN, Hesselson SE, Zhan Y, Aquino C, Cao Y, et al. Next generation genome-wide association tool: design and coverage of a highthroughput European-optimized SNP array. Genomics. 2011;98:79-89.

76. Saccone SF, Bierut LJ, Chesler EJ, Kalivas PW, Lerman C, Saccone NL, et al. Supplementing high-density SNP microarrays for additional coverage of disease-related genes: addiction as a paradigm. PLoS One. 2009;4:e5225.

77. Saccone SF, Saccone NL, Swan GE, Madden PAF, Goate AM, Rice JP, et al. Systematic biological prioritization after a genome-wide association study: an application to nicotine dependence. Bioinformatics. 2008;24:1805-11.

78. Ashburner M, Ball CA, Blake JA, Botstein D, Butler H, Michael Cherry J, et al. Gene Ontology: tool for the unification of biology. Nat Genet. 2000;25:25-9.

79. IPA, QIAGEN, Redwood City, CA. http://www.qiagen.com/ingenuity. Accessed 20 Nov 2015.

80. Nakajima M, Yamamoto T, Nunoya K, Yokoi T, Nagashima K, Inoue K, et al. Role of human cytochrome P4502A6 in C-oxidation of nicotine. Drug Metab Dispos. 1996;24:1212-7.

81. Nakajima M, Yamamoto T, Nunoya K, Yokoi T, Nagashima K, Inoue K, et al. Characterization of CYP2A6 involved in 3'-hydroxylation of cotinine in human liver microsomes. J Pharmacol Exp Ther. 1996;277:1010-5.

82. Bloom AJ, Martinez M, Chen L-S, Bierut LJ, Murphy SE, Goate A. CYP2B6 Non-Coding Variation Associated with Smoking Cessation Is Also Associated with Differences in Allelic Expression, Splicing, and Nicotine Metabolism Independent of Common Amino-Acid Changes. PLoS One. 2013;8:e79700.

83. Hukkanen J, Jacob 3rd P, Benowitz NL. Metabolism and disposition kinetics of nicotine. Pharmacol Rev. 2005;57:79-115. 
84. Jarvik ME, Madsen DC, Olmstead RE, Iwamoto-Schaap PN, Elins JL, Benowitz NL. Nicotine blood levels and subjective craving for cigarettes. Pharmacol Biochem Behav. 2000;66:553-8.

85. Saccone SF, Hinrichs AL, Saccone NL, Chase GA, Konvicka K, Madden PA et al. Cholinergic nicotinic receptor genes implicated in a nicotine dependence association study targeting 348 candidate genes with 3713 SNPs. Hum Mol Genet. 2007;16:36-49.

86. Saccone NL, Schwantes-An T-H, Wang JC, Grucza RA, Breslau N, Hatsukami $D$, et al. Multiple cholinergic nicotinic receptor genes affect nicotine dependence risk in African and European Americans. Genes Brain Behav. 2010;9:741-50

87. Hancock DB, Wang J-C, Gaddis NC, Levy JL, Saccone NL, Stitzel JA, et al. A multiancestry study identifies novel genetic associations with CHRNA5 methylation in human brain and risk of nicotine dependence. Hum Mol Genet. 2015;24:5940-54

88. Hancock DB, Reginsson GW, Gaddis NC, Chen X, Saccone NL, Lutz SM, et al. Genome-wide meta-analysis reveals common splice site acceptor variant in CHRNA4 associated with nicotine dependence. Transl Psychiatry. 2015;5:e651.

89. Exome Variant Server, NHLBI GO Exome Sequencing Project (ESP), Seattle, WA. http://evs.gs.washington.edu/evs/. Accessed 1 Sept 2013.

90. Browning SR, Browning BL. Rapid and accurate haplotype phasing and missing-data inference for whole-genome association studies by use of localized haplotype clustering. Am J Hum Genet. 2007;81:1084-97.

91. R Core Team. R: A language and environment for statistical computing. Vienna, Austria; R Foundation for Statistical Computing; 2014. http://www.Rproject.org/. Accessed 20 Nov 2015

\section{Submit your next manuscript to BioMed Central} and we will help you at every step:

- We accept pre-submission inquiries

- Our selector tool helps you to find the most relevant journal

- We provide round the clock customer support

- Convenient online submission

- Thorough peer review

- Inclusion in PubMed and all major indexing services

- Maximum visibility for your research

Submit your manuscript at www.biomedcentral.com/submit 